Supporting Information for:

\title{
Versatile Solid Phase Synthesis of Gold Nanoparticle Dimers Using an Asymmetric Functionalization Approach
}

\author{
Rajesh Sardar, Tyler Heap, and Jennifer S. Shumaker-Parry* \\ Department of Chemistry, University of Utah \\ 315 S. 1400 E. Salt Lake City, Utah 84112
}

\section{Experimental Procedures:}

Materials and Methods. All materials were used as received without further purification. Chloroauric acid $(\mathrm{HAuCl} 4 \bullet 3 \mathrm{H} 2 \mathrm{O})$, trisodium citrate, 16mercaptohexadecanoic acid (MHA), mercaptoethylamine (MEA), 11-mercapto-1undecanol (MUOH), 1-pentafluorophenol (PFP), 1-ethyl-3-(3-dimethylaminopropyl)carbodiimide hydrochloride (EDAC) were obtained from Aldrich.

Experimental Techniques. Absorption spectra were recorded using a Perkin-Elmer Lamda 9 UV/Vis/NIR spectrophotometer. Transmission electron microscopy (TEM) measurements were carried out using a Tecnai T-12 TEM at $120 \mathrm{kV}$ operating voltage. Sonication was done using a Branson ultrasonic cleaner (Model no. 2510).

Synthesis of Gold Nanoparticles. Gold colloids were prepared by sodium citrate reduction method of $\mathrm{HAuCl}_{4}$ (Aldrich) reported by Turkevich, et al. ${ }^{1}$ All glassware was cleaned with aqua-regia (3:1 $\left.\mathrm{HCl}: \mathrm{HNO}_{3}\right)$, rinsed with MilliQ water, and dried in an oven at $120{ }^{\circ} \mathrm{C}$ over night before use. In a typical procedure, $190 \mathrm{~mL}$ distilled water $(>18 \mathrm{M} \Omega$ ) mixed with $10 \mathrm{~mL}$ of $2 \mathrm{~g} / \mathrm{mL} \mathrm{HAuCl} 4$ was brought to a vigorous boil while being stirred rapidly in a $500 \mathrm{~mL}$ round bottom flask fitted with a reflux condenser. When the solution started to boil, $2 \mathrm{~mL}$ of $10 \mathrm{mg} / \mathrm{mL}$ trisodium citrate solution in water was added while heating and stirring continued. This resulted in the solution changing to a deep red color within 20 minutes. At this point, the dispersion was removed from the heat and allowed to cool to room temperature. The nanoparticle dispersion was filtered through a $0.2 \mu \mathrm{M}$ filter paper to remove large aggregates. The diameters of the particles were determined by TEM analysis. The TEM sample was prepared by placing a drop of gold nanoparticle suspension on a formvar-coated copper grid and excess solution was removed by wicking using a filter paper to avoid particle aggregation. The grid was then allowed to dry before it was imaged. The nanoparticle diameters were determined by analysis of TEM images counting 150 particles using NIH image software, ImageJ. Employing a similar method, gold nanoparticles with different sizes were prepared by varying the molar concentration of trisodium citrate. 
Functionalization of Glass Surfaces with 3-Aminopropyltriethoxy-silane and Subsequent Adsorption of Gold Nanoparticles. Glass coverslips were functionalized with 3-aminopropyltriethoxy-silane using the published procedure. ${ }^{2}$ The coverslips were cleaned by placing them in an aqueous $20 \%$ RBS detergent solution heated to $90{ }^{\circ} \mathrm{C}$ and sonicating for 5 minutes. The coverslips were copiously rinsed with distilled water and then immersed in a $1: 1(\mathrm{v} / \mathrm{v})$ solution of methanol and concentrated $\mathrm{HCl}$ for 30 minutes. The coverslips were rinsed thoroughly with distilled water and left to dry overnight in an oven at $60{ }^{\circ} \mathrm{C}$. The clean coverslips were then placed in a $10 \%(\mathrm{v} / \mathrm{v})$ solution of (3aminopropyl)triethoxysilane in anhydrous ethanol for 30 minutes. The coverslips were sonicated and then rinsed with anhydrous ethanol and dried at $120{ }^{\circ} \mathrm{C}$ for $3 \mathrm{~h}$. Finally, the silanized glass coverslips were immersed in aqueous gold nanoparticle solution for $24 \mathrm{~h}$ at room temperature.

Functionalization of AuNPs with MUOH. A self-assembled monolayer of MUOH was formed on the surface of the gold nanoparticles by incubation in a $1 \mathrm{mM}$ solution of $\mathrm{MUOH}$ in ethanol for $2 \mathrm{~h}$ at room temperature. After formation of the MUOH SAMs, the surface was thoroughly rinsed with ethanol to remove unreacted thiols, dried using nitrogen, and stored at $4{ }^{\circ} \mathrm{C}$ in a $\mathrm{N}_{2}$ atmosphere.

Functionalization of MUOH-Capped AuNPs with MHA or MEA. The MUOH functionalized AuNPs adsorbed on the silanized glass surface were sonicated in $5 \mathrm{ml}$ of ethanol containing $50 \mu \mathrm{l} 1 \mathrm{mM}$ of 16-mercaptohexadecanoic acid (MHA) in ethanol for 5 minutes. A similar process was followed, substituting MEA for MUOH, to obtain MEAfunctionalized gold nanoparticles.

Synthesis of Gold Nanoparticles Dimers. The partially MHA functionalized AuNPs were then reacted with $100 \mu \mathrm{l}$ of $0.1 \mathrm{M}$ EDAC and $100 \mu \mathrm{l}$ of $0.2 \mathrm{M}$ PFP for $2 \mathrm{~h}$ at room temperature followed by the addition of MEA-functionalized gold nanoparticles. The reaction mixture was stirred gently at room temperature for $4 \mathrm{~h}$ under a nitrogen atmosphere. The dimer solution showed remarkable stability over a period of two months after storage at $4{ }^{0} \mathrm{C}$ under a nitrogen atmosphere.

Surface Plasmon Resonance (SPR) Characterization Using UV-visible Absorption Spectroscopy. UV-visible absorption spectra were collected for citrate-stabilized Au NPs in water and partially-functionalized (MUOH, MHA, and MEA) particles in ethanol. For SPR analysis of particles immobilized on glass, the surrounding dielectric environment was air. 


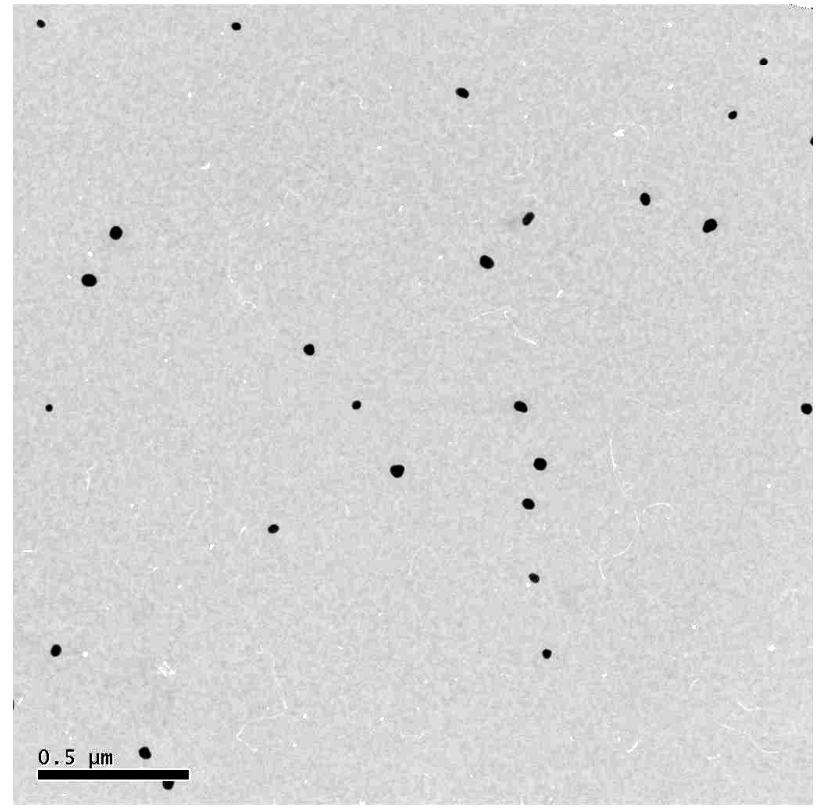

Figure S1: TEM image of Partially MHA-functionalized 41-nm-diameter AuNPs.

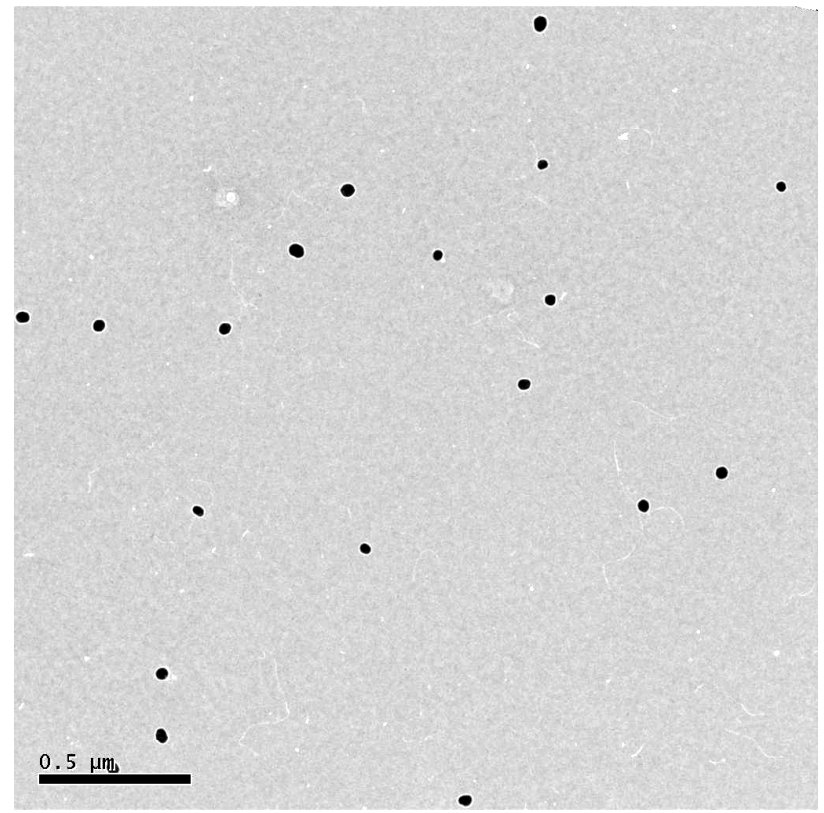

Figure S2: TEM image of partially MEA-functionalized 41-nm-diameter AuNPs. 


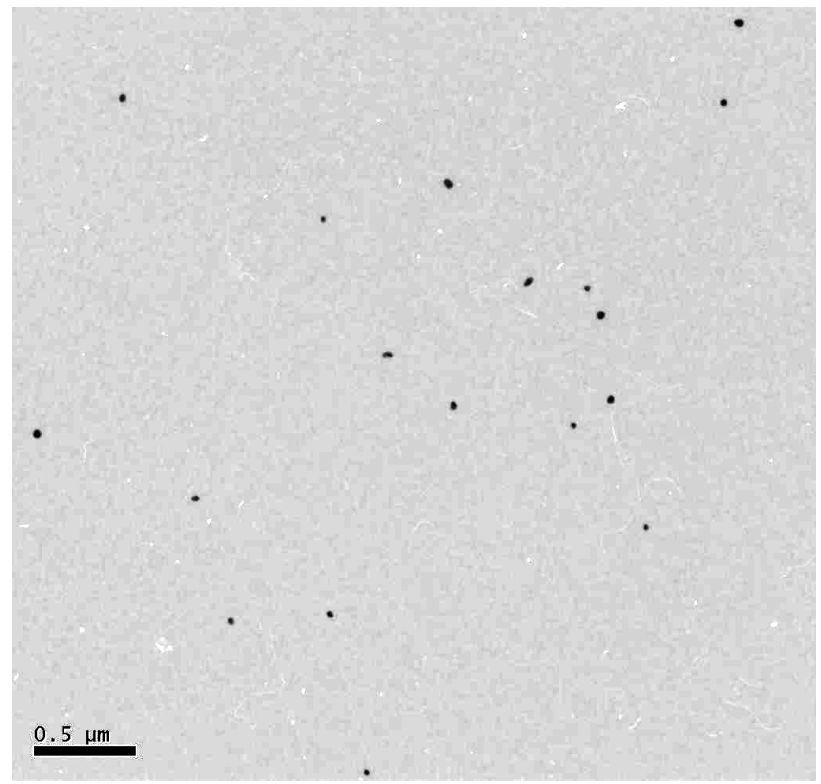

Figure S3: TEM image of control experiment of 40-nm-diameter gold nanoparticles functionalized with MHA and MEA without coupling agents EDAC and PFP.

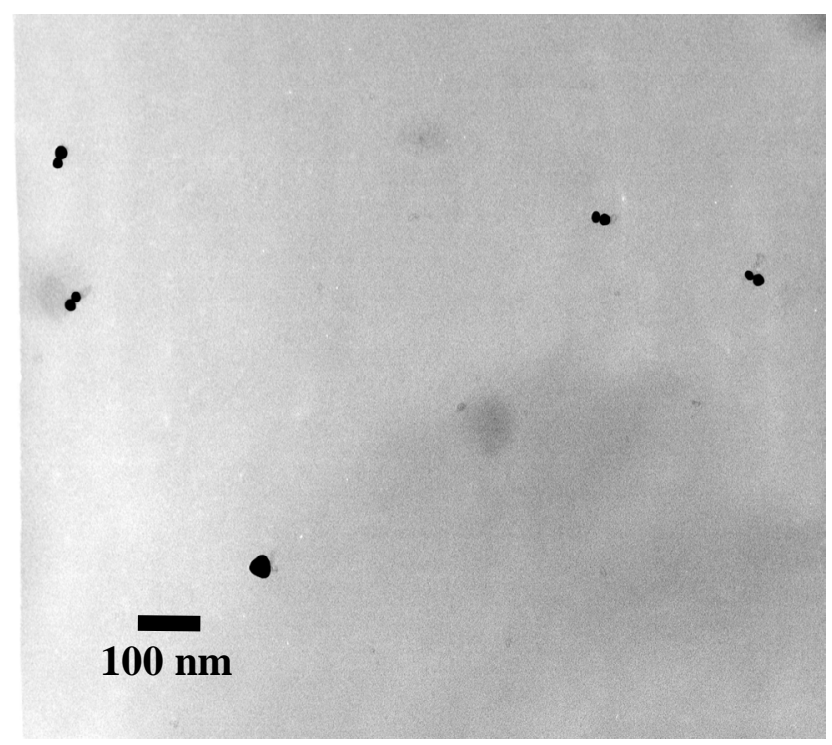

Figure S4: TEM image of heterodimers synthesized from coupling 30- and 16-nmdiameter gold particles. Scale bar is $100 \mathrm{~nm}$ 


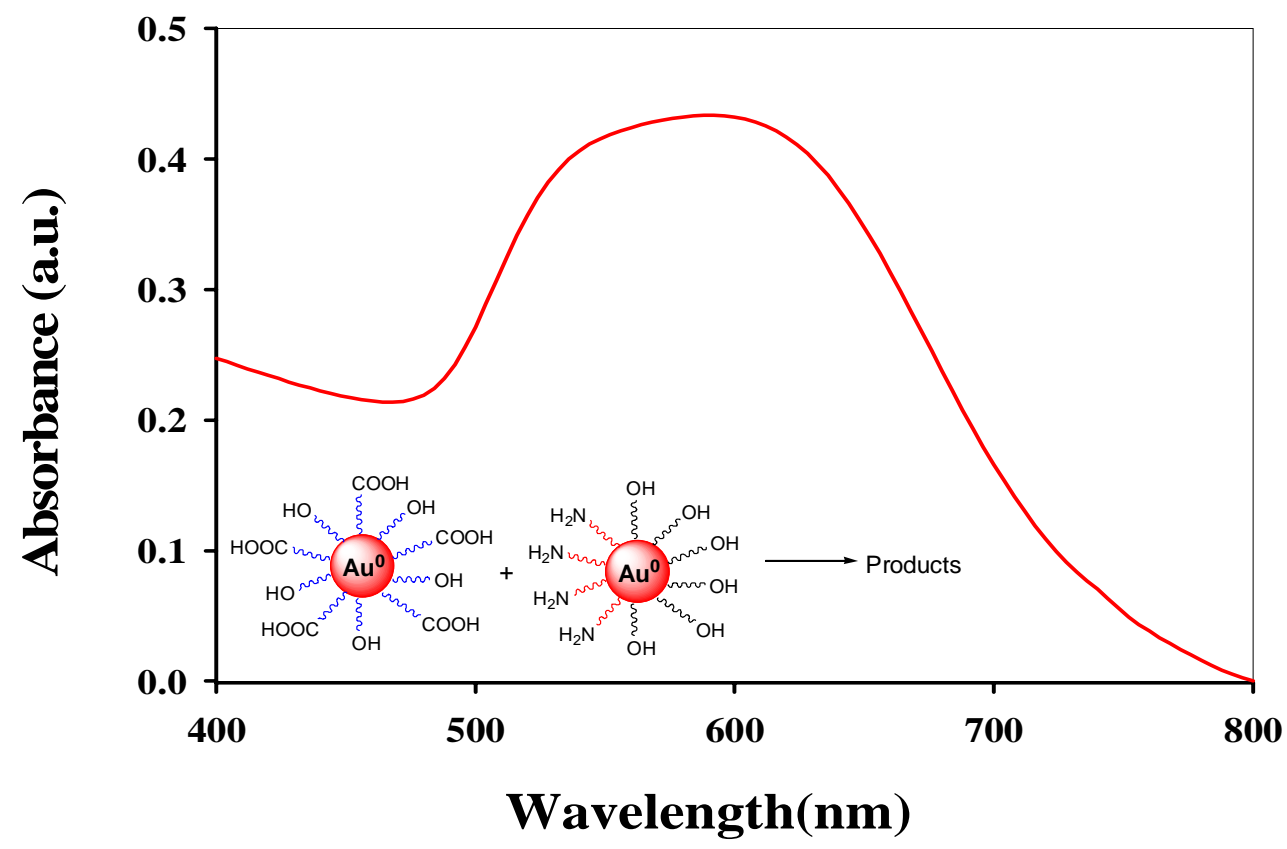

Figure S5: UV-Vis absorption spectrum of the products when 50:50: MUOH:MHA functionalized AuNPs were reacted with partially amine-functionalized particles.

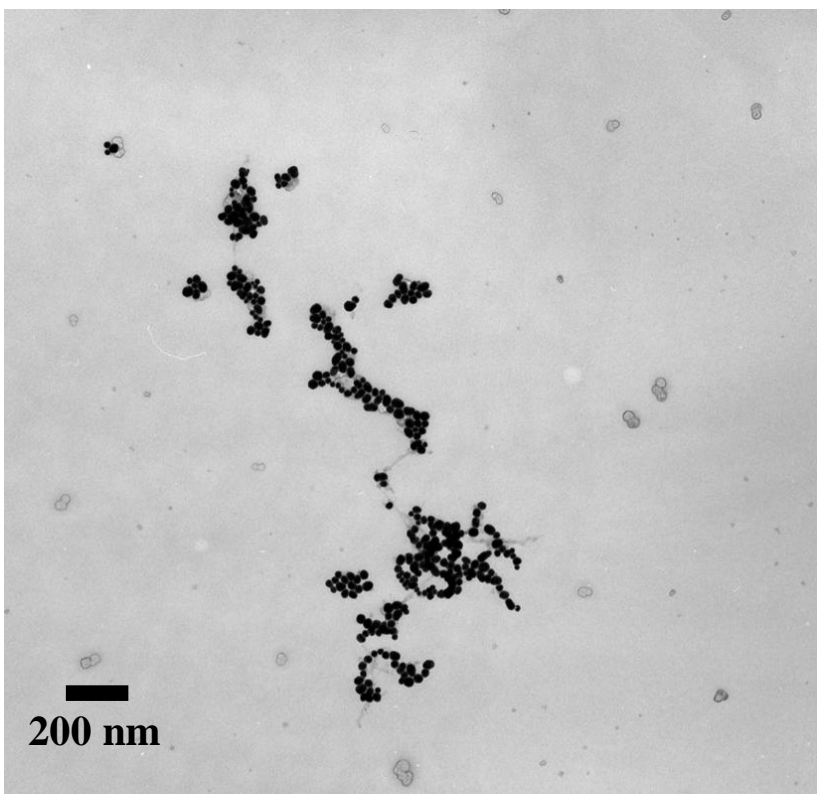

Figure S6: TEM image of the products when 50:50: MUOH:MHA functionalized AuNPs were reacted with partially amine functionalized particles. 


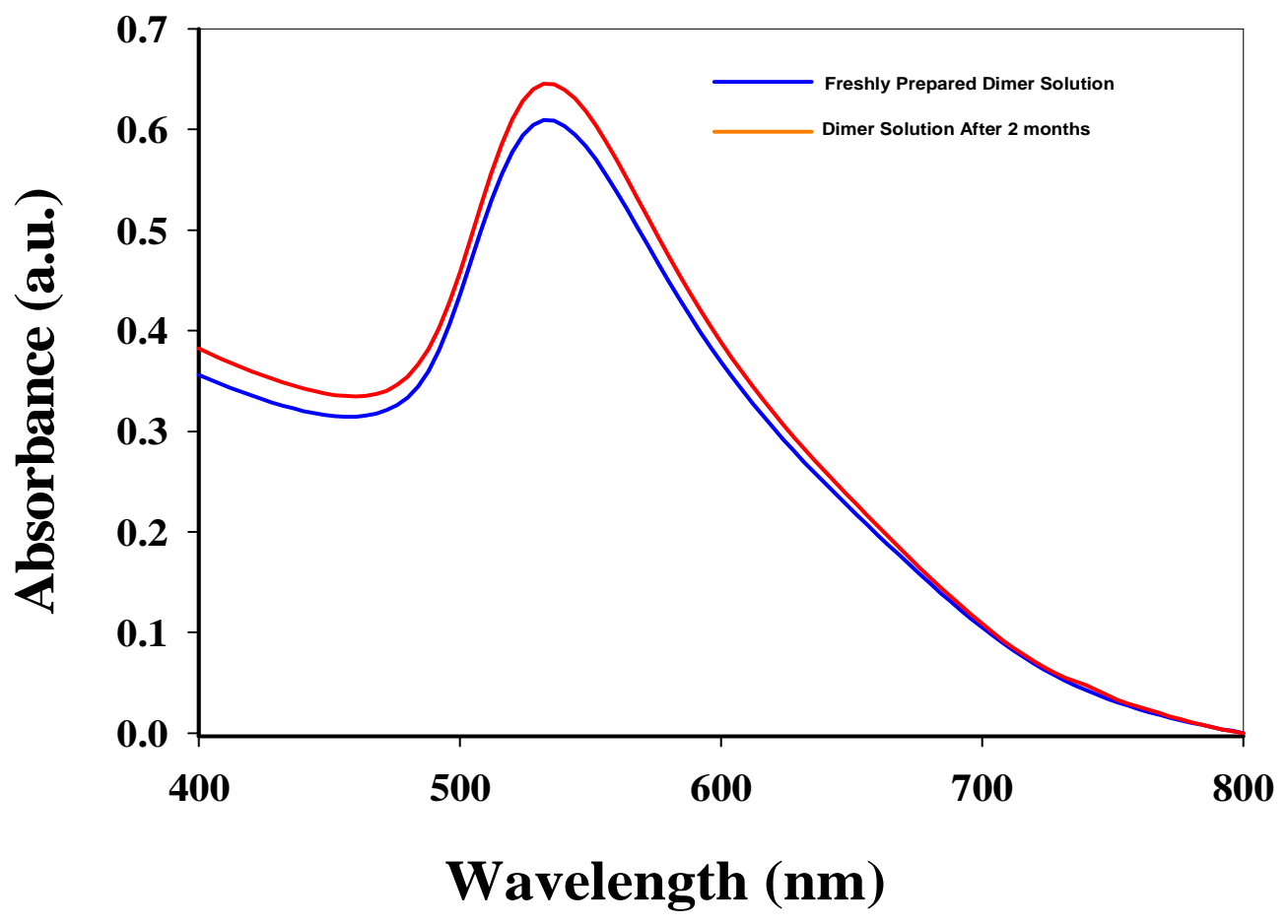

Figure S6: UV-visible absorption spectra comparison of freshly prepared dimer solution and solution after 2 months.

\section{Reference.}

1. Turkevich, J.; Stevenson, P. C.; Hillier, J. Discuss. Faraday Soc. 1951, 11, 55-75

2. Nath, N.; Chilkoti, A. Anal. Chem. 2004, 76, 5370-5378. 